\title{
7 In eigener Sache: das Berufsbild „Unternehmensbibliothekar für digitale Services“
}

Im Schlusskapitel möchte ich Sie einladen, über das veränderte Berufsbild im digitalen Umfeld, sowie einige erforderliche Kompetenzen im Unternehmen und mögliche Entwicklungen nachzudenken. Wir wünschen uns alle Orientierung, wie es mit uns Bibliothekaren, speziell auch in den Unternehmen, weitergehen könnte. Wir haben es ja einerseits mit internen Umstrukturierungen, Automatisierung, Outsourcing zu tun und andererseits mit den Entwicklungen der Bibliotheksdienstleistungen und Verlage im Internet - dabei kann man das Gefühl haben, dass die alten Dienste nicht mehr gebraucht werden. Wenn wir genauer hinschauen, sehen wir aber, dass viel Neues hinzugekommen ist, was uns bereichert und hilft, unsere Arbeit zu unterstützen. Es gilt, gerade im digitalen Unternehmensumfeld die eigene Rolle neu zu finden.

\subsection{Der Job-Titel und was dahinter stecken könnte}

Das Image des Bibliothekars wird im Allgemeinen mit den Tätigkeiten der Kollegen aus öffentlichen Einrichtungen assoziiert. Ebenso mit der physischen Bibliothek selbst, den gedruckten Medien, den Räumlichkeiten oder dem Gebäude - also dem

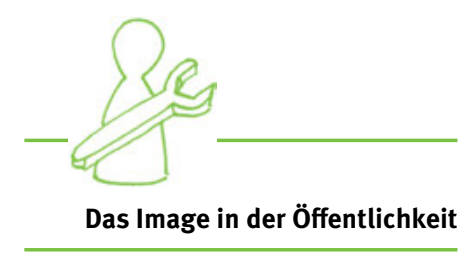
klassischen Modell. Dass es auch in Unternehmen Informationsvermittlungsstellen oder Bibliotheken gibt, die sich dezentral und virtuell um das Management von Fachliteratur für das Unternehmen an verschiedenen Standorten kümmern könnten, ist Außenstehenden oft nicht bekannt. Schon gar nicht, welche Aufgaben die Arbeit der Unternehmensbibliothekare beinhalten kann. Mag sein, dass es daran liegt, dass das Berufsbild dahinter keine feste, klare Bezeichnung hat, die in der Öffentlichkeit wahrgenommen wird. Das liegt sicher auch daran, dass die Tätigkeiten so speziell sind. Unternehmensbibliotheken sind sogenannte Spezialbibliotheken. Die Berufsbezeichnung „Spezialbibliothekar“ ist zwar inhaltlich richtig und die Tätigkeiten in der bibliothekarischen Welt weitestgehend geläufig, aber darüber hinaus kaum bekannt (vgl. Behm-Steidel). Deshalb verwendet unsere Berufsgruppe häufig die Bezeichnung „Information Professional“, um damit zu zeigen, dass wir „Informationen ernst nehmen“ (vgl. Motto der DGI) und Aufgaben erledigen, die inhaltlich mit den Schnittmengen zwischen „Bibliothek und Information“ oder weiter gefasst auch mit „Wissensmanagement“ zu tun haben können. Aber auch diese Bezeichnung dürfte über die ABD und IuD-Grenzen hinaus eher Ratlosigkeit als ein Aha-Erlebnis hervorrufen.

Mitarbeitern und Entscheidern in Unternehmen wird es vermutlich ähnlich gehen. Sie sind auch ein Querschnitt der Gesellschaft und können oft nicht einschätzen, wie wertvoll die Zusammenarbeit mit der eigenen Unternehmensbibliothek sein könnte. Diese Herausforderung ist nicht neu. Der in diesem Buch verwendete Begriff „Unternehmensbibliothekar“ ist deshalb eher ein Kunstbegriff und selten zu finden. Er soll aber verdeutlichen, dass diese Tätigkeit spezielle, für dieses Arbeitsfeld benötigte Kompetenzen erfordert. Eine transparente Gestaltung und Kommunikation über die eigenen Tätigkeiten ist von daher umso wichtiger.

Im digitalen Umfeld treten die Services der Bibliothek aus dem physischen Umfeld heraus und werden zunehmend auf den Bildschirmen der internen Kunden präsent. Die Mitarbeiter stellen ihre Fragen einer Suchmaschine oder Datenbank, anstatt sich an ihre Bibliothek zu wenden. Die Tätigkeit des Bibliothekars besteht in der Gestaltung elektronischer Dienstleistungen. Dadurch entstehen neue Rollen und Berufsbezeichnungen, die nichts mehr mit dem klassischen Modell zu tun haben. In Un- 

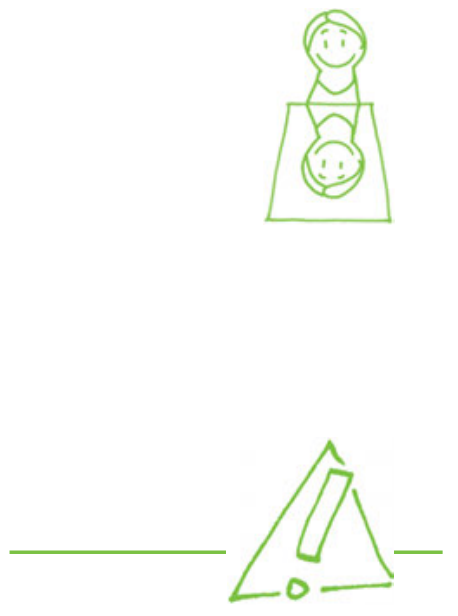

Bibliothekare sind Generalisten

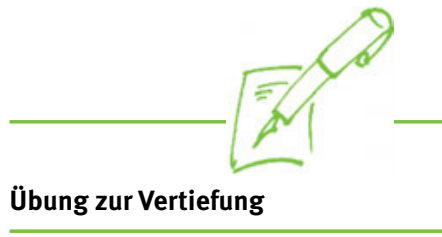

Übung zur Vertiefung ternehmen werden für diese Rollen oft Bezeichnungen vergeben, die etwas beschreiben, was „irgendwie“ mit Literatur, Information, Spezialwissen, und den Tätigkeiten des Organisierens und Managens zu tun haben.

\section{Übung zum Selbsttest:}

Es gibt im Internet „Job-Titel-Generatoren“, die Ihnen bei jedem Klick eine wohlklingende Berufsbezeichnung anbieten. Hier unten ist ein Beispiel aus der Bibliothekswelt. Nach ein paar Klicks werden Sie sich im Bürostuhl verwundert aufrichten. Nach weiteren Klicks werden Sie lächeln, denn Sie haben eine Kurzerkenntnis: „Ja, stimmt“, werden Sie denken, „das mache ich auch alles“. Genau - das ist der Trick und schon mal ein guter Anfang - obwohl die Bezeichnungen auf Englisch sind. Machen Sie einmal den Test, Sie werden viel Spaß haben!

http://www.michellemach.com/jobtitles/index.html (Stand: 28.04.2014)

Denken Sie sich ein paar weitere Titel aus und schauen Sie, was diese für Assoziationen bei Ihnen wecken: Informationsmanager, Internet Librarian, Teaching Librarian, Systembibliothekar ...

Im Unternehmen sind Bibliothekare Generalisten, die eine Mischung aus vielen Tätigkeiten ausüben. Laut Duden ist ein Generalist jemand, der in seinen Interessen nicht auf ein bestimmtes Gebiet festgelegt ist. Wie dynamisch bibiothekarische Themenfelder in methodische Bereiche der IT oder des Managements vordringen, ist sicher deutlich geworden. Deshalb ist fast alles, was uns der „Job-Titel-Generator“ ausspuckt, auch irgendwie richtig. Darin mag einerseits die Chance liegen, unsere Vielfältigkeit darzustellen, andererseits aber unser Hauptproblem, dass wir das Gefühl haben könnten, keine Bezeichnung zu finden, die eindeutig oder konkret genug ist.

Als Bibliothekare sind wir gerade im Unternehmen von Experten umgeben, unsere Kundschaft ist meist gut ausgebildet, Akademiker mit einem komplexen Fachstudium, Manager mit viel Verantwortung. Da kann man sich, vor allem, wenn man als Solist arbeitet, schnell als „Exot“ fühlen. Dabei sind Unternehmensbibliothekare, wie alle anderen Berufsgruppen auch, Mitarbeiter und damit Teil des Unternehmens. Sie können mit ihrem methodischen Wissen über die Organisation von Information aktiv zum Geschehen im Unternehmen beitragen und haben eine breite Vernetzung, weil sie in der Regel Dienstleistungen für das ganze Unternehmen erbringen können. Als Grenzgänger zwischen den Funktionen entsteht ein Blick für viele Informationsbedürfnisse und damit ein vernetztes Denken, da Unternehmensbibliothekare von vornherein zwischen Anbietern, Kunden und Managementebenen agieren.

\section{Vertiefung zur eigenen Sichtweise des Berufsbildes:}

Gehen wir davon aus, Sie als Leser sind als Bibliothekar oder im weitesten Sinne mit Literatur verwaltenden Aufgaben in einem Unternehmen beschäftigt. Fragen Sie nach, was andere über das Berufsbild denken und was sie sich unter Ihrer Tätigkeit vorstellen.

- Was antworten Sie, wenn Sie nach Ihrem Beruf oder Ihrer Tätigkeit gefragt werden?

- Wie sind die Reaktionen?

- Wie beschreiben Sie, was Sie tun?

- Welche Begriffe verwenden Sie? 


\subsection{Erforderliche Kompetenzen}

Da Unternehmensbibliothekare oft in kleinen Teams arbeiten, müssen viele Aufgaben und dazu noch Projekte gleichzeitig übernommen werden. Die Grenze zwischen den rein fachlichen Aufgaben des Bibliothekars und der Verantwortung eines Managers ist oftmals fließend, gerade, wenn häufig in Projektteams gearbeitet wird. Hinzu kommt, dass Unternehmensbibliothekare auch Personalverantwortung übernehmen oder sich der Ausbildung von Azubis und Praktikanten widmen. Dabei kann man sich schon wie ein Jongleur fühlen, der viele Bälle in der Luft halten muss.

Der Unternehmensbibliothekar zeigt mit seiner Profession Wertschätzung für die Fachinformation. Der Sinn vieler seiner Aufgaben liegt ja gerade darin, dass mit Hilfe von Qualitätskriterien „Wissen“ strukturiert wird und man dazu beitragen kann, Filter gegen die Informationsüberflutung zu bieten. Im digitalen Zeitalter ist dies keineswegs überholt, sondern eigentlich zwingend notwendig. Somit sorgt man indirekt für eine der Grundlagen, auf denen das Unternehmen Entscheidungen trifft, auch wenn dies nicht immer unmittelbar so gesehen wird. Ein gut organisierter Service kann ein konkurrenzfähiger Vorteil für wissensintensive Unternehmen sein. Deshalb ist es so wichtig, dass Trends in der Unternehmens- und Informationswelt ständig dahingehend überprüft werden, ob sie einen Einfluss auf die eigene Zielsetzung haben.

Die Herausforderungen, die sich aus dem digitalen Wandel ergeben, sind in Kapitel 2 bereits dargestellt worden. Die Auflistung der Kernaufgaben finden Sie in Kapitel 2.2.2. Welche Kompetenzen uns die Special Libraries Association (SLA) für das 21. Jahrhundert darüber hinaus empfiehlt, deckt sich vielfach mit der Einschätzung anderer Autoren. Methodisch konzentriert man sich hauptsächlich auf Kompetenzen, die vor allem den Umgang mit publizierten Fachinformationen betreffen. Fassen wir hier noch einmal kurz zusammen:

- Beschaffung von Fachinformationen zum Nutzen des Unternehmens,

- Management von elektronischen und gedruckten Informationsressourcen,

- kontinuierliche Evaluierung des Nutzens sowie Vor- und Nachteile gedruckter und elektronischer Versionen,

- Kenntnis über Formate, Zugriffsbedingungen und Konditionen von Online-Publikationen,

- Kenntnis und Kontakte zu Verlagen, Produzenten, Providern,

- Verhandlungen von Konditionen,

- Organisation eines reibungslosen Services, der dem internen Kunden ermöglicht, selbständig und schnell Fachinformationen zu finden und zu nutzen,

- Entwicklung von Verhaltensregeln beim Umgang mit externen Informationsressourcen.

- Überprüfung der Wirtschaftlichkeit der genutzten Ressourcen durch die Entwicklung geeigneter Kennzahlen.

IT-Kompetenzen sind bis zu einem gewissen Grad notwendig, um Prozesse und Anwendungssysteme aus dem Blickwinkel des Nutzers und administrativen Anwenders zu verstehen - es reicht nicht aus, nur Begeisterung für die Möglichkeiten der Technik zu zeigen. Der Info-Profi muss in der Lage sein, elektronische Serviceabläufe zu konzipieren, zu evaluieren und zu testen. Darüber hinaus agiert er als Bindeglied zwischen Technik und Mensch, in dem er wie ein Berater einzuschätzen versucht, was die Tools können, welchen Nutzen sie bringen und ob die internen Kunden damit zurechtkommen. Er organisiert die Vernetzung der Tools und muss dafür ein syste-

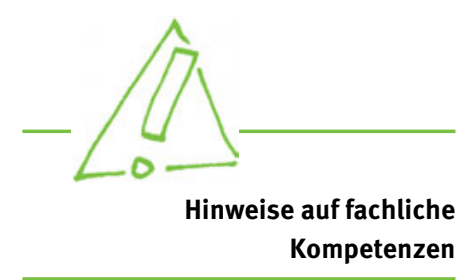




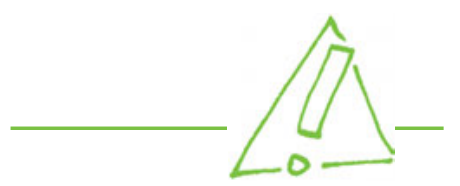

Hinweise auf soziale Kompetenzen misch-analytisches Denken mitbringen. Darüber hinaus vertritt er als eine Art „Anwalt" die Kundeninteressen gegenüber den Anbietern.

Kommunikative und kooperative Kompetenzen sind ebenfalls erforderlich, wenn ein professioneller Umgang mit anderen Funktionen und unternehmensinternen Berufsgruppen ausgeübt wird. Unter Schnittstellenkompetenz versteht man, die internen Rollen zu kennen und mit ihnen zusammen zu arbeiten. Die geeigneten Methoden sind Audits, Interviews, Runde Tische und andere Gremien. Kommunikation macht vermutlich $80 \%$ der Tätigkeit aus. Trotz Social Media ist der Bedarf an sinnvoller Kommunikation über digital verfügbare Information erst recht notwendig geworden. Der Unternehmensbibliothekar entwickelt Kundenschulungen zum richtigen Umgang mit den Dienstleistungen. Er entwickelt Compliance-Regeln zum richtigen Umgang mit Daten und konzipiert Veranstaltungen, um beide Ziele zu erreichen. Bei Routinetätigkeiten ist vor allem Geduld erforderlich, die gleichen Fragen von verschiedenen Kunden immer wieder neu zu beantworten. Vielleicht sogar, die eigentliche Frage hinter einem Problem zu erkennen, auch wenn dies nur indirekt ausgedrückt wird.

Wenn man aufmerksam Stellenanzeigen liest, scheint sich der Eindruck zu bestätigen, dass eine Zunahme von „Schlüsselqualifikationen“ oder sozialen Kompetenzen, wie selbständiges Arbeiten, Verantwortungsbereitschaft, besonderes Engagement und Serviceorientierung verlangt werden. Durch die zunehmende Automatisierung standardisierbarer Abläufe wird sich der Fokus vermehrt auf diese Schlüsselqualifikationen verlagern und noch wichtiger werden.

Eine der wichtigsten Eigenschaften im Unternehmen, die aber selten explizit genannt wird, ist wohl die Flexibilität. Gemeint ist, sich in einer ständig wandelnden Umwelt in Abstimmung mit allen Beteiligten operativ immer wieder an strategische Veränderungen anzupassen. Prioritäten können sich kurzfristig ändern, dass heißt, gut laufende Prozesse und Services können plötzlich wegfallen und etwas ganz anderes gefragt sein. Ein Umdenken ist nicht immer einfach. Deshalb muss man kreativ und innovativ bleiben, um lösungsorientierte Ideen zu entwickeln. Diese dürfen gern pragmatisch sein und müssen dann oft in Eigeninitiative entstehen. Dafür ist es notwendig, mit anderen Kollegen zu „netzwerkeln“, um sich Input zu holen.

\section{Vertiefung:}

Die eben dargestellten Anforderungen, muss jeder für sich selbst entwickeln. Erkennen Sie sich hier und da wieder? Machen Sie eine kleine Übung. Ab und zu kann ein kleiner Selbsttest nicht schaden. Stellen Sie sich hin und wieder folgende Fragen sowie auch die Antworten dazu vor. Wenn Sie mutig sind, können Sie zur Überprüfung auch Ihre Kollegen um Feedback bitten. Lassen Sie sich überraschen:

Wie würden die Kollegen Sie beschreiben? 


\subsection{Evaluierung der eigenen Wirksamkeit}

Kennzahlen zu entwickeln, um die Leistungsfähigkeit der Services zu untermauern, ist wichtig. Deshalb enthalten auch die meisten Kapitel Hinweise hierzu. Aber die Wirksamkeit oder den Impact der eigenen Maßnahmen zu evaluieren, ist etwas, das weit darüber hinausgeht (vgl. Dueck). Was wir hier selbst entwickeln müssen, sind Methoden, die das implizite Wirken der Gesamtheit der Services stärker verdeutlicht. Das ist leichter gesagt als getan. Woran wird die Wirksamkeit einer Dienstleistung gemessen?

Wenn wir von Wirkung sprechen, brauchen wir zunächst eine Ursache. Das kann tatsächlich die Nachfrage oder die Dienstleistung selbst sein. Deshalb sollte man hier ansetzen und sich folgende Fragen immer wieder stellen.

- Was wird getan?

- Für wen?

- $\mathrm{Zu}$ welchen Konditionen?

- Mit welchen Methoden?

Dabei ist auch gefragt, sich in die Sichtweisen des Umfeldes hineinzudenken. Greifen Sie Ihre Analysen von den FAQs und Vertiefungen aus Kapitel 1.2 auf (Positionierung innerhalb des Unternehmens) und versuchen Sie, diese um die hier genannten Fragestellungen zu ergänzen. Wirksamkeit zu entdecken ist ein laufender Prozess, dem mehr Aufmerksamkeit geschenkt werden muss. Der Denkprozess beginnt mit der Re- 


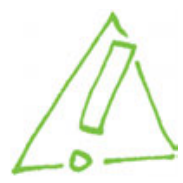

flexion über die eigenen Rollen, die Aufgaben, die digitalen Veränderungen und die ständigen Herausforderungen. Man muss sogar bereit sein, je nach Antwort, seine Prioritäten zu ändern.

Für eine Evaluierung wäre es empfehlenswert, das ganze Bibliotheksteam einzubinden, weil alle Feedback von ihren Kunden zu den Services erhalten (vgl. Shumaker). Im Virtuellen wird das Wirken unsichtbar. Das Team sollte ein gemeinsames Verständnis für die Notwendigkeit entwickeln, seine Wirksamkeit zu entdecken, sichtbar zu machen und konkret zu untermauern und sich für die Beschreibung der Wirksamkeit Kriterien auszudenken, die im Kontext des Unternehmens vorkommen und dort verstanden werden.

Fragen Sie sich regelmäßig:

- Was war der konkrete Nutzen der eigenen Arbeit?

- Haben Sie Ihre Zielsetzung erreicht?

- Bieten Sie den richtigen Service an?

- Evaluieren Sie an der richtigen Stelle?

- Sind die Kriterien richtig definiert?

Suchen Sie nach Möglichkeiten, diesen Fragen auf die Spur zu kommen. Hier muss wieder die Bibliothek zum Kunden gehen. Feedback versteckt sich nämlich auch in informellen Anekdoten oder bei alltäglichen Problemlösungen. Wenn der Nutzen der eigenen Dienstleistung durch positive Rückmeldungen bestätigt wurde, sammeln Sie Beispiele und kommunizieren Sie diese in regelmäßigen Berichten an Kunden und Management. Vielleicht können diese Beispiele dazu beitragen, ein Bild von der Durchdringung der digitalen Services der Unternehmensbibliothek innerhalb des Unternehmen zu verdeutlichen. Sammeln Sie zusätzlich Fakten, die die Stärken der Abteilung, sowie finanzielle Vorteile oder Kostenersparnis des vorhandenen Services verdeutlichen.

- Beinhalten Ihre Services Aspekte von Schnelligkeit, Zeitersparnis?

- Wo wurden Kosten eingespart?

- Wo haben sich im Vergleich zum klassischen Modell sichtbare Vorteile durch den Nutzen von digitalen Serviceabläufen ergeben?

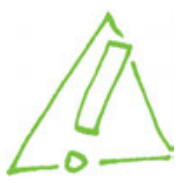

In kleineren Unternehmen wird vieles aufgrund der flachen Hierarchien in direkter Interaktion mit den Arbeitskollegen stattfinden. In größeren Unternehmen braucht man für die Umsetzung dieser Strategie Unterstützung von oben. Dies ist ein permanenter Prozess.

- Pflegen Sie deshalb Beziehungen zu allen Managementebenen,

- $\quad$ sorgen Sie für eine Wahrnehmung des eigenen Beitrages zum Nutzen des Unternehmens

\subsection{Qualifikationsmöglichkeiten oder „learning on the job“?}

Wo und wie kann man dies alles lernen? Wo kann man Zertifikate erwerben? Gibt es überhaupt Möglichkeiten, die Kompetenzen, die man im Laufe seines Berufslebens schon entwickelt hat, besser zum Ausdruck zu bringen? Eine schwierige Frage. Bereitet einen die Ausbildung oder das Studium auf bestimmte inhaltliche Qualifikationen vor und bringt uns darüber hinaus die Basis des Methodenwissens bei, so muss man feststellen, dass vieles „by the way“, „on the fly“ und ,in between“ passiert. Der Arbeitsalltag ist dicht gedrängt und für Fortbildungen wird wenig Zeit bleiben. Das Lernen selbst findet während der laufenden Arbeit statt. Viele Unternehmen erwarten, 
dass man sich selbst einen routinierten Umgang mit neuen Programmen, Tools und Software aneignet oder durch Schulungen seine Kenntnisse erweitert. Vieles findet durch direkten Austausch mit Kollegen statt. Hier kann man die schon mehrfach angesprochene Beziehung zur IT-Abteilung nutzen, um ein Verständnis für den Aufbau der eigenen Infrastruktur zu entwickeln. Manche Unternehmen veranstalten eigene Weiterbildungen. Versuchen Sie, sich daran zu orientieren und selbständig zu lernen.

Eine Mitarbeiterentwicklung wird sicher in vielen Unternehmen stattfinden. Sollten sich Ihre Tätigkeiten mit zunehmend digitalen Angeboten verändert haben, so ist es hin und wieder ratsam, seine Stellenbeschreibungen zu aktualisieren. Nicht immer denkt man im Arbeitsalltag an diese Aspekte. Sprechen Sie offen mit Ihrem Vorgesetzten die Veränderungen an, die sich ergeben. Vor allem dann, wenn er ein „fachfremder“ Kollege ist. Erkundigen Sie sich nach seiner Sichtweise. Jährliche Zielerreichungsgespräche sind vielleicht ein guter Anlass, gezielte Schulungen zu vereinbaren. Tauschen Sie sich auch mit Kollegen darüber aus.

Sind mehr und mehr strategische Qualifikationen gefragt, so kann man diese in speziellen Seminaren erwerben, die Methoden im Projektmanagement, Innovations- oder Qualitätsmanagement vermitteln. Auch eine Grundlagenvermittlung von Präsentationstechniken, Kommunikation und PR können hilfreich sein, seine Ziele zu erreichen. Wie in Kapitel 4 erwähnt, sind auch praktische Fähigkeiten, wie richtiges Verhandeln wichtig. Diese kann man sich beispielsweise in Lehrgängen für den Bereich Einkauf aneignen. Kaufmännische Grundlagen sind ebenfalls nicht unwichtig und können zum Beispiel in Lehrgängen verschiedener IHKs erworben werden.

Darüber hinaus können Kenntnisse der Mitarbeiterführung gefragt sein, die ebenfalls über Seminare vermittelt werden. Einzelne Universitäten oder Fachhochschulen, die Bachelor- und Masterstudiengänge anbieten, haben auch für Berufspraktiker teilweise ein interessantes Programm (nachzulesen auf den Webseiten). Berufsverbände bieten ebenfalls Tipps für Seminare oder gar eigene Fortbildungen, auch im Rahmen ihrer Tagungen an und geben sicher gerne Auskunft. Wenn man sich eingehender mit dem Urheberrecht beschäftigen will, so sind die Verwertungsgesellschaften eine gute Quelle. Es gibt immer wieder Publikationen zu dem Thema sowie gezielte Veranstaltungen. Interessant ist hier die seit einigen Jahren stattfindende Urheberrechtstagung in Göttingen, veranstaltet von zwei juristischen Lehrstühlen und der Universitätsbibliothek Göttingen. Sie wendet sich an alle am Thema Interessierten und hat den Anspruch, nicht nur eine rein juristische Veranstaltung zu sein.

Veranstaltungen von fachfremden Seminaranbietern können erfrischend sein, weil sie eine andere Sprache sprechen und neue Sichtweisen einbringen. Ob man E-Learning-Konzepte mag, ist Typfrage. Man kann diese am Arbeitsplatz ausführen, wird aber auf einen persönlichen Gedankenaustausch mit anderen Seminarteilnehmern verzichten müssen.

Wichtig ist das kontinuierliche Studium von Fachliteratur (Zeitschriften und Fachbücher), sowie das Abonnieren von Mailinglisten und Newslettern zu einzelnen Interessensgebieten, um Trends in der Informationsvermittlung zu erkennen. Einige Tipps werden Sie im Anhang der Internetlinks finden.

Die Interaktion mit Berufskollegen aus anderen Branchen darf nicht unterschätzt werden. Grob gesagt fällt diese Aktivität unter das „netzwerkeln“, und bedeutet konkret Erfahrungsaustausch. Man kann auf diese Weise gut voneinander lernen und profitieren. Der Besuch von Fachtagungen dient nicht nur dazu, sich Vorträge zu bestimmten Themen anzuhören. Oftmals kann man das Gehörte ja nicht immer eins zu eins im eigenen Betrieb anwenden, erfährt aber von Problemlösungsstrategien, die man vielleicht in die eigene Arbeit transferieren kann. Dabei sollten nicht nur bibliotheksspezifische Tagungen, sondern auch Veranstaltungen aus der Informationswis-

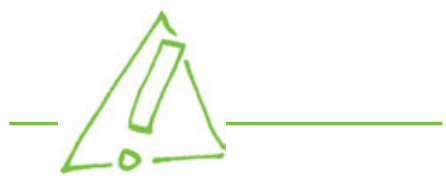

Stellenbeschreibung anpassen

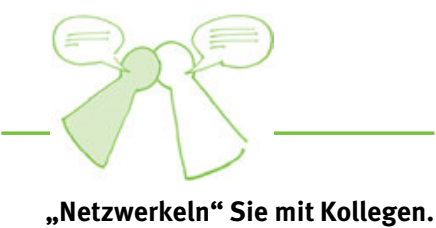



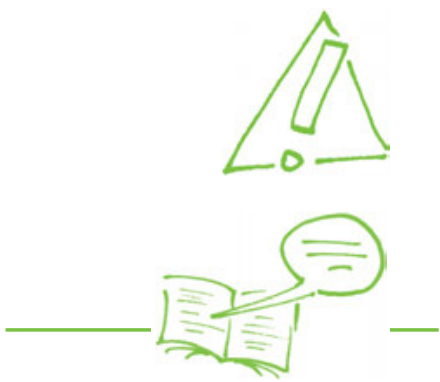

„Wenn die Maschinen, die die Menschen so im Laufe der Zeit erfunden haben, nun auch noch funktionierten: was wäre das für ein angenehmes Leben.“ (Kurt Tucholsky)

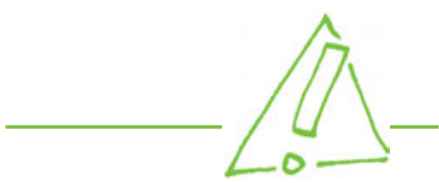

„Klappern gehört zum Handwerk!“ senschaft und dem Wissensmanagement besucht werden, weil sich in der täglichen Arbeit ohnehin Überschneidungen zu diesen Fachbereichen ergeben. Gerade jungen Kollegen sei es empfohlen, sich Berufsverbänden und Fachgesellschaften anzuschließen, vielleicht sogar aktiv in einem Arbeitskreis mitzuarbeiten. Nicht immer ist der unmittelbare Nutzen dieser Mitgliedschaften gleich erkennbar, aber mittelbar entstehen Beziehungen, die einem vielleicht irgendwann weiterhelfen können - oder umgekehrt. Wie schon erwähnt bieten Buch- und ähnliche Fachmessen Einblicke in Entwicklungen bei Providern und Agenturen. Manche Provider organisieren eigene Info-Veranstaltungen in den großen Ballungszentren.

Die maßgeschneiderten Fortbildungen für alle Aspekte Ihres Berufsalltages wird es nicht geben. Bleiben Sie neugierig, schauen Sie über den Tellerrand und haben Sie Spaß daran, neue Sichtweisen und Erkenntnisse zu gewinnen.

\subsection{Fazit}

Diskussionen über die Entwicklung des Berufsbildes sind alles andere als neu und sie werden zweifellos mit der fortschreitenden Digitalisierung und technologischen Entwicklung fortgeführt. Das neben stehende Zitat von Kurt Tucholsky könnte uns einstweilen beruhigen, dass uns die Arbeit so schnell nicht ausgehen wird...

Vielleicht kommt es im Internet- und Wissenszeitalter mehr auf Kompetenzen an, die Projekte und Services „zum Gelingen“ zu bringen. Reines Fachwissen und reine Fachkenntnisse werden in Zukunft alleine nicht mehr ausreichen. Weil der Trend weitergeht, standardisierbare Tätigkeiten zu automatisieren, wird es künftig mehr darum gehen, als Unternehmensbibliothek einen Mehrwert zu bieten, der für die Kunden maßgeschneiderte Lösungen für das Arbeiten mit digitalen Quellen bietet. Darauf werden wir uns einstellen müssen. Dabei werden wir uns mit unterschiedlichen Professionen zunehmend vernetzen und in ständiger Kommunikation bleiben müssen.

Wir Info-Profis sollten ein bisschen Selbstmarketing betreiben, damit der Wert, der Anteil und die Wirksamkeit unserer Leistung besser verstanden werden. Klappern gehört zum Handwerk - klappern Sie mit! Wir scheuen uns vielleicht vor dem Gedanken, aber das, was unser Berufsstand zum Thema Selbstdarstellung gelegentlich als Übertreibung empfinden mag, ist es für andere keineswegs. Machen Sie auf Ihre spezielle Expertise aufmerksam.

\section{Literaturhinweise zu Kapitel 7:}

Bauschmann, Martin: Jung, dynamisch, berufserfahren...: ein Längsschnittvergleich von Stellenausschreibungen der Bibliotheks- und Informationsbranche. - Berlin: BibSpider, 2012. - (Wiborada - Leipziger Schriften zur Bibliotheks- und Informationswissenschaft; 3)

Behm-Steidel, Gudrun: Kompetenzen für Spezialbibliothekare : eine Untersuchung zu Anforderungen und Qualifizierung von Beschäftigten in internen Informationseinrichtungen. - Berlin: Logos, 2001. - (Berliner Arbeiten zur Bibliothekswissenschaft ; 6)

Competencies for Information Professionals of the 21st Century/Special Libraries Association (SLA). Revised edition, June 2003 http://www.sla.org/about-sla/competencies/ (Stand: 22.04.2014)

Dueck, Gunter: Professionelle Intelligenz : worauf es morgen ankommt. - 1. Aufl. - Frankfurt a.M.: Eichborn, 2011

Gelungene Expansion von Business Intelligence in Sekundär- und qualitative Forschung: ein erfolgreicher Information Professional verfügt über umfassende Informationskompetenz und ist in 
der Kundenkommunikation erfahren / [Interview von Password mit Dr. Sabine Graumann von TNS Infratest (München)]. - In: Password (2013), Nr. 2, S. 16 ff.

Hales, Stuart: New Opportunities for Information Professionals. - In: Information Outlook, Vol. 17 (2013) Nr. 2, S. 8-9

Poetzsch, Eleonore: Lehre und Forschung in der Information und Dokumentation: eine Bilanz über mehr als drei Jahrzehnte: zum Stand unserer Profession. - In: Password (2013), Nr. 6, S. 6 ff.

Renn, Oliver: Alles nur Job-Title-Bingo - oder wie können Information Professionals die Innovations kraft ihres Unternehmens unterstützen? - Vortrag auf den 2. DGI-Praxistagen „Faktor ,i‘- Strategischer Umgang mit Informationen in Unternehmen“, Frankfurt a. M., 08.-09.11.2012. - 26 S. (PDF-Präsentation)

http://www.dgi-info.de/images/Veranstaltungen/Renn_2.DGI-Praxistage_121109.pdf

(Stand: 22.04.2014)

Shumaker, David: The Embedded Librarian: Innovative Strategies for Taking Knowledge Where It's Needed. - Medford, NJ: Information Today, 2012 